\title{
An Old Disease With an Unfamiliar Face: A Case Report of Ocular Syphilis
}

\author{
George Boghdadia , Max Feldman ${ }^{\mathrm{b}, \mathrm{c}}$
}

\begin{abstract}
Syphilis is an infection caused by the bacterium Treponema pallidum. Patients with syphilis can present with a wide range of symptoms depending on the stage of disease. An underappreciated manifestation of syphilis is ocular involvement, which has been increasingly reported in the last several years. Delay in diagnosis and treatment of ocular syphilis can increase the risk of irreversible vision loss. Syphilis can affect almost every structure in the eye; however, most commonly presents with panuveitis. Ocular syphilis is treated similarly to neurosyphilis with prompt initiation of intravenous penicillin $\mathrm{G}$.
\end{abstract}

Keywords: Ocular syphilis; Neurosyphilis; Uveitis

\section{Introduction}

An underappreciated manifestation of syphilis is ocular involvement, which has been increasingly reported in the last several years. Delay in diagnosis and treatment can increase the risk of long-term vision loss. We present a case of a 40-year-old female with panuveitis due to syphilis infection.

\section{Case Report}

A 40-year-old female presented to her ophthalmologist's office for evaluation of decreased visual acuity and bilateral flashers. She was diagnosed with bilateral panuveitis, started on cyclopentolate and prednisolone drops, and was referred to her primary care physician for further evaluation. Upon questioning, the patient also reported a pruritic rash of 3 weeks duration on both lower extremities and a genital ulcer 6 weeks prior that

\footnotetext{
Manuscript submitted February 13, 2020, accepted March 2, 2020
}

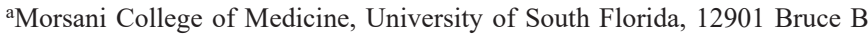
Downs Blvd, Tampa, FL 33612, USA

${ }^{b}$ Department of Internal Medicine, Morsani College of Medicine, University of South Florida, 12901 Bruce B Downs Blvd, Tampa, FL 33612, USA

${ }^{\mathrm{c} C}$ Corresponding Author: Max Feldman, Department of Internal Medicine, Morsani College of Medicine, 12901 Bruce B Downs Blvd, Tampa, FL 33612, USA. Email: mfeldma1@health.usf.edu

doi: https://doi.org/10.14740/jmc3438 had since resolved.

She otherwise felt well with no complaints. The patient denied arthralgias, oral ulcers, photosensitivity, chest pain, or shortness of breath. She had no known exposures to tuberculosis. She was sexually active with one male partner, however questioned if he was monogamous. She had no prior history of sexually transmitted infections and never had been screened for human immunodeficiency virus (HIV). She had a past medical history of hypertension that was well controlled on amlodipine and was otherwise in excellent health. She had no family history of systemic autoimmune diseases or sarcoidosis.

Her physical exam was notable for copper colored/hyperpigmented macules on bilateral feet and ankles with overlying xerotic patches as shown in Figure 1.

Rapid plasma reagin (RPR) was positive with a 1:64 titer and her treponemal antibody was reactive. HIV screen was negative. The patient was diagnosed with secondary syphilis and was admitted to the hospital for evaluation of neurosyphilis given concurrent uveitis. Upon admission she underwent a lumbar puncture (LP) with results as follows: two nucleated cells; 0 red blood cells (RBCs) (100\% polymorphonuclears (PMNs)); protein 23, glucose 60; cerebrospinal fluid (CSF) Venereal Disease Research Laboratory (VDRL) non-reactive; herpes simplex virus (HSV) IgG and IgM negative.

The rash was biopsied which showed superficial and deep

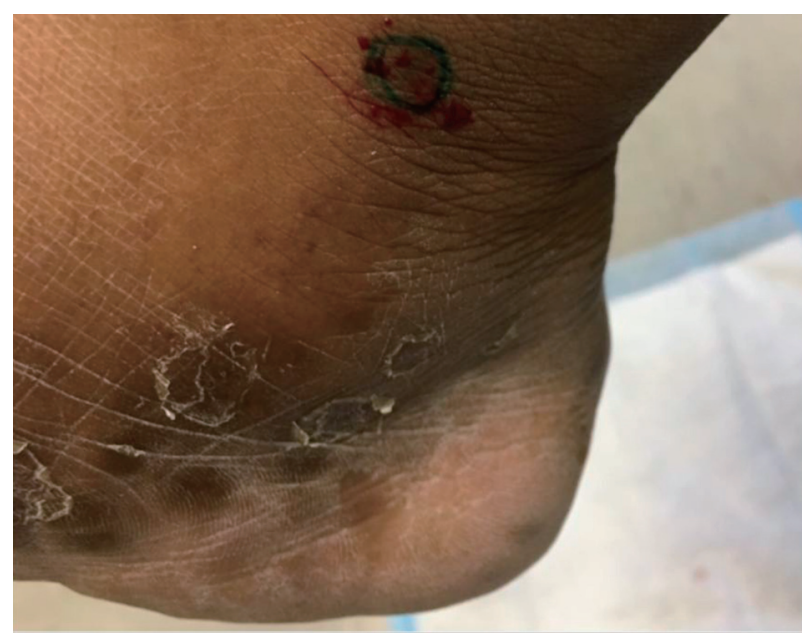

Figure 1. Lower extremity rash. Copper colored/hyperpigmented macules on ankle with overlying xerotic patches. Biopsy showed superficial and deep perivascular dermatitis with treponemal organisms consistent with cutaneous syphilis. 
perivascular dermatitis with treponemal organisms consistent with cutaneous syphilis. Ophthalmologic exam revealed an inferior retinal hole in the left eye and bilateral panuveitis. Infectious disease was consulted and recommended 14 days of IV penicillin. Ophthalmology recommended concurrent use of topical cyclopentolate $1 \%$ and prednisolone $1 \%$. The patient tolerated the treatment course well and had complete resolution of her rash and uveitis, without any long-term effects on visual acuity. As CSF findings were normal, no repeat LP was performed. Follow-up RPR titers checked at 6 months decreased to $1: 32$.

\section{Discussion}

True to its nickname, "the great masquerader" has been reported to affect almost every structure in the eye and can mimic other infectious and immune ocular inflammatory disorders [1]. Panuveitis is the most common manifestation of ocular syphilis and usually presents with eye pain, vision loss, flashers/floaters, and photophobia [2]. Ocular syphilis can occur and overlap with any stage of syphilis infection; however, eye involvement occurs most frequently in secondary syphilis and late syphilis [1]. Diagnosis is usually presumptive based on the presence of inflammatory eye changes and positive serologies.

Ocular syphilis is distinct from neurosyphilis and can occur with or without other neurologic symptoms or CSF changes. All patients with ocular syphilis should receive an LP even in the absence of neurological symptoms to evaluate for neurosyphilis [3]. Up to $70 \%$ of patients with ocular syphilis will also have evidence of neurosyphilis on CSF testing [1].

Ocular syphilis should be treated the same as neurosyphilis even if the LP is normal. The recommended regimen is aqueous crystalline penicillin G 3-4 million units IV every $4 \mathrm{~h}$ or continuous infusion for 10 - 14 days [3]. If the LP is positive, patients will need serial LPs to ensure proper response to therapy [3].

Of note, the duration of therapy for ocular syphilis is shorter than the course needed for treatment of tertiary and late latent syphilis, therefore one should consider an additional dose of intramuscular (IM) penicillin G after completion of IV penicillin for those patients with ocular manifestations during these states [3]. Management of ocular syphilis should be done in consultation with infectious disease and ophthalmology.

Ocular syphilis should be considered on the differential of any inflammatory eye disorder. When suspected, the diagnosis requires urgent workup including LP and ophthalmologic exam, along with prompt initiation of antibiotics. Most patients will have complete recovery; however, delay in treatment can result in serious sequala including blindness [1].

\section{Acknowledgments}

None to declare.

\section{Financial Disclosure}

None to declare.

\section{Conflict of Interest}

None to declare.

\section{Informed Consent}

Informed consent from the patient was obtained for this case report.

\section{Author Contributions}

George Boghdadi contributed to the literature review and drafting of the manuscript. Max Feldman contributed to the project conception, literature review, drafting, critical revision and final approval of the manuscript.

\section{Data Availability}

Any inquiries regarding supporting data availability of this study should be directed to the corresponding author.

\section{References}

1. Oliver SE, Aubin M, Atwell L, Matthias J, Cope A, Mobley V, Goode A, et al. Ocular Syphilis - Eight Jurisdictions, United States, 2014-2015. MMWR Morb Mortal Wkly Rep. 2016;65(43):1185-1188.

2. Moradi A, Salek S, Daniel E, Gangaputra S, Ostheimer TA, Burkholder BM, Leung TG, et al. Clinical features and incidence rates of ocular complications in patients with ocular syphilis. Am J Ophthalmol. 2015;159(2):334343 e331.

3. Workowski KA, Bolan GA. Sexually transmitted diseases treatment guidelines, 2015. MMWR Recomm Rep. 2015;64(RR-03):1-137. 